\title{
Strict control of glycaemia: effects on blood flow in the large retinal vessels and in the macular microcirculation
}

Juan E Grunwald, Charles E Riva, Benno L Petrig, Alexander J Brucker, Stanley S Schwartz, Seth N Braunstein, Joan DuPont, Sharon Grunwald

\begin{abstract}
Aims-The purpose of this study was to investigate the effect of instituting strict diabetic glycaemic control on the retinal macular microcirculation and to compare this effect with that observed in the main retinal veins.

Methods-In 28 insulin dependent diabetic patients with poor glycaemic control a regimen of strict diabetic control, consisting of four daily insulin injections was instituted and maintained for 6 months. Retinal haemodynamics were investigated in the macular microcirculation by the blue field simulation technique and in the major retinal veins by a combination of bidirectional laser Doppler velocimetry and monochromatic fundus photography. Progression of diabetic retinopathy was assessed from fundus photographs taken at baseline and at the end of the study.
\end{abstract}

Results-Institution of strict diabetic control resulted in a significant increase in leucocyte velocity in the macular circulation $(p=0 \cdot 013)$. No significant difference in this increase was observed between eyes that showed progression $(n=8)$ and no progression $(n=20)$ of retinopathy during the study. Significant correlations were found between relative changes over time of blood flow measured in the main retinal veins and relative changes of leucocyte velocity determined in the macular microcirculation at 2 months $(p=0.008)$ and 6 months $(p=0.001)$ but not at 5 days $(p=0 \cdot 49)$. In the eight eyes that showed progression of retinopathy, the product of leucocyte velocity and density at baseline was significantly higher than normal $(p<0.05)$. During the length of this study, this product was also significantly higher in the eight eyes that showed retinopathy progression than in the 20 eyes that did not show progression $(p=0 \cdot 005)$.

Conclusion-Our results suggest that increased flow in the macular microcirculation may be associated with progression of retinopathy, thus supporting the hypothesis that increased blood flow may play a role in the development of diabetic microangiopathy. Although there are correlations between the changes detected in the macular microcirculation and those measured in the main retinal vessels, there are also differences which need to be further investigated in order to better understand pathogenetic mechanisms. (Br f Ophthalmol 1995; 79: 735-741)

Long term strict control of glycaemia has been shown to slow down the development and progression of diabetic retinopathy. ${ }^{1}$ Shortly after the institution of strict diabetic control, however, some patients show progression of diabetic retinopathy. ${ }^{1-7}$ Using bidirectional laser Doppler velocimetry (BLDV) and monochromatic fundus photography (MFP), we have previously demonstrated that institution of strict diabetic control induces changes in the circulation of the main retinal vessels. ${ }^{89}$ Decreases in retinal blood flow occurring soon after the institution of strict diabetic control are associated with no progression of retinopathy. Patients who have progression of retinopathy do not show such decreases in flow after the institution of strict diabetic control.

In this report, we investigate the effect of strict diabetic control on the macular circulation, as assessed by the blue field simulation technique (BFS). In addition, we include data on the relation between blood flow in the macular capillaries, as measured by BFS, and that in the main retinal vessels, as assessed by BLDV and MFP.

\section{Methods}

Twenty eight insulin dependent diabetic patients aged between 18 and 43 years (mean 31 (SD 6) years) with disease duration of 5 to 28 years (16 (7) years) were investigated. All patients had non-proliferative diabetic retinopathy and poor diabetic glycaemic control, as demonstrated by glycosylated haemoglobin levels higher than $3 \mathrm{SD}$ above the mean of normal subjects. Other inclusion and exclusion criteria have been described in details elsewhere. ${ }^{9}$ A baseline BFS measurement was obtained in these patients and also in 16 normal subjects with no history of systemic or ocular eye disease and a normal eye examination. Ages of these normal volunteers ranged from 23 to 49 years (32 (8) years).

BLUE FIELD SIMULATION MEASUREMENTS

Leucocyte velocity in the macular retinal capillaries and leucocyte number in a $20^{\circ}$ region centred at the foveola can be determined quantitatively using the BFS technique. ${ }^{1011}$ 
This technique is based on the observation of the blue field entoptic phenomenon.

The blue field entoptic phenomenon can be seen with highest contrast when the retina is uniformly illuminated with diffuse light at a wavelength of $430 \mathrm{~nm}$. Under these conditions, a large number of leucocytes are observed entoptically as dot-like luminous particles with a darker tail moving along curved paths in a pulsatile fashion. These normally invisible paths correspond to the macular retinal capillary bed.

Since the velocity and density (number of particles within the field of view) of these entoptically observed leucocytes cannot be measured directly, the BFS technique offers an indirect approach to quantify these variables. A field of simulated moving particles is generated by computer and presented on a screen. The density and velocity of the simulated leucocytes can be adjusted by means of dials on a control panel.

The ability to match leucocyte velocity accurately was tested in each subject before the start of the study. Subjects were asked to adjust the velocity of simulated particles in one computer simulated field, $V_{1}$, to match that in a second simulated field, $V_{2}$, which was set at a fixed value. From the mean and standard deviation of 5 adjustments of $\mathrm{V}_{1}$ we determined the expected adjustment error: $\mathrm{E}=100 \%$ (mean $\left.\mathrm{V}_{1}-\mathrm{V}_{2}\right) / \mathrm{V}_{2}$. Subjects who could not achieve $\mathrm{E}$ values smaller than $25 \%$ were not included in this study. Average E for the patients was $9 \%$ (SD $6 \%$ ).

Subjects were then asked to observe alternately the entoptic phenomenon and the computer simulation, and to adjust the velocity (Vleuc) and density (Dens, number of particles within the field of view) of the simulated leucocytes until they matched those of the entoptically observed leucocytes. The computer then recorded the subject's simulation screen settings and stored the results on file. To reduce the effect of adjustment variability on the measurements, these adjustments were repeated five times and the average and the standard deviation for each of the adjusted variables were determined. Between consecutive adjustment trials, the position of the velocity dial was scrambled by adding a random offset to the physical dial position. The position of the Dens dial was not scrambled. Average coefficients of variation for baseline Vleuc measurements were $21 \%(8 \%)$ in diabetic patients and $23 \%(10 \%)$ in normal subjects. Light intensity levels that permitted an optimal visualisation of the blue field entoptic phenomenon, and were below $12 \mu \mathrm{W} / \mathrm{cm}^{2}$, were used.

BIDIRECTIONAL LASER DOPPLER VELOCIMETRY MEASUREMENTS

A Polaroid colour fundus photograph of the posterior fundus was obtained for documentation of the sites of bidirectional laser Doppler velocimetry (BLDV) measurements. BLDV measurements of the maximum or centreline erythrocyte velocity $\left(V_{\max }\right)$ were obtained in main superior and inferior temporal retinal veins. The location of the measurement site was marked on the Polaroid photograph for reference.

Fundus photographs were taken in monochromatic light at $570 \mathrm{~nm}$. From these, the venous diameter (D) at the site of BLDV measurement was determined from projected photographic negatives. D corresponds to an average of the measurements obtained from six photographs. All measurements of $\mathrm{D}$ were performed by one trained examiner, and all $\mathrm{V}_{\max }$ determinations were done by another examiner. Each examiner was masked with regard to the results of the other examiner, status of diabetic control, and status of diabetic retinopathy.

Immediately after blood flow measurements, intraocular pressure (IOP) was measured by applanation tonometry, and brachial artery blood pressure was obtained by sphygmomanometry.

Volumetric blood flow rate $(Q)$ was calculated as described previously ${ }^{12}$ as $\mathrm{Q}=\mathrm{V}_{\text {mean }} \pi$ $\mathrm{D}^{2} / 4$, where mean blood velocity $\left(\mathrm{V}_{\text {mean }}\right)$ was calculated as $\mathrm{V}_{\text {mean }}=\mathrm{CV}_{\text {max }}$. A value of $\mathrm{C}$ equal to $1 / 1.6$ was used, and the relation between $\mathrm{V}_{\max }$ and $\mathrm{V}_{\text {mean }}$ was assumed to remain unchanged during the study.

During each session, BFS, BLDV, and MFP measurements were obtained during room air breathing and $100 \%$ oxygen breathing. Following baseline measurements, subjects breathed $100 \%$ oxygen through a mouthpiece. Breathing through the nose was prevented by a nose clip. BLDV measurements were obtained between $3 \frac{1}{2}$ and $5 \frac{1}{2}$ minutes of oxygen breathing and MFP was obtained immediately thereafter. BFS determinations were performed on a separate experiment at about 3 to 8 minutes of $100 \%$ oxygen breathing.

The regulatory response to $100 \%$ oxygen breathing, characterised as the percentage change in leucocyte velocity $\left(R_{\text {Vleuc }}\right)$ during oxygen breathing, was calculated using the formula $\quad R_{\text {Vleuc }}=100 \% \quad\left(\right.$ Vleuc $_{\text {air }}-$ Vleuc $\left._{\mathrm{O} 2}\right) /$ Vleuc $_{\text {air }}$. A similar formula was used to calculate the regulatory response to $100 \%$ oxygen breathing of Vleuc $\times$ Dens $\left(R_{\text {Vleuc } \times \text { Dens }}\right)$ and $Q\left(R_{Q}\right)$.

All BFS and BLDV measurements were performed in one eye only, chosen at random at the beginning of the study. The sequence of BFS or BLDV measurements was randomly chosen at the beginning of the study. For each patient the same order was maintained during the length of the study. A 30 minute rest was given to the subjects between BFS and BLDV measurements. Pupils were dilated with $1 \%$ tropicamide and $10 \%$ phenylephrine hydrochloride before BFS and BLDV measurements. The BFS measurements were done through a $4 \mathrm{~mm}$ diameter artificial pupil. Blood glucose was measured with an AccuCheck blood glucose monitor (Boehringer Mannheim, Indianapolis, IN, USA) immediately after the BFS and BLDV measurements.

STUDY PROTOCOL

Patients had a first session of baseline blood flow measurement. A second baseline blood flow measurement was performed 3-4 weeks 

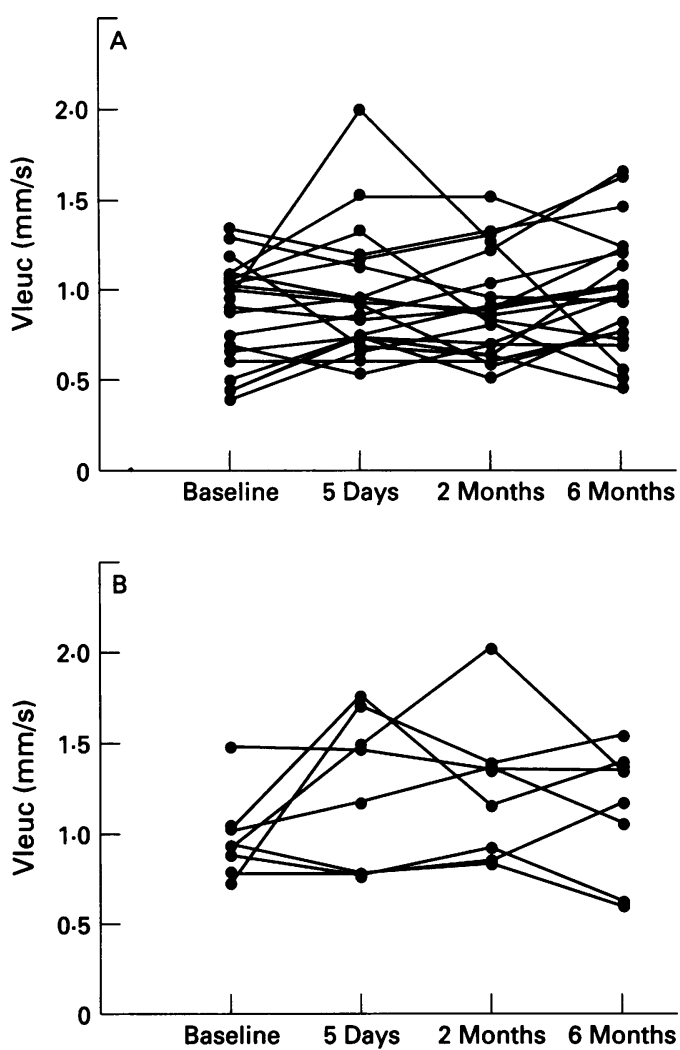

Figure 1 Leucocyte velocity (Vleuc) in the retinal macular circulation at baseline and then 5 days, 2 months, and 6 months after the institution of strict glycemic control. (A) Eyes that showed no progression of retinopathy at the end of the 6 month study. (B) Eyes that showed progression of retinopathy at the end of the study.

later. Immediately thereafter patients were hospitalised and a regimen of strict diabetic control of blood glucose similar to that used in the Diabetic Control and Complications Trial (DCCT) ${ }^{13}$ was instituted under careful monitoring. Patients were asked to monitor blood glucose levels (finger capillary blood samples determined by an Accu-Check blood glucose monitor) four times daily before their meals and at bedtime. Patients self administered four daily insulin injections with a goal of achieving blood glucose levels within the following guidelines: preprandial, 3.9-6.7 $\mathrm{mM} ; 2 \mathrm{~h}$ postprandial, $<8.4 \mathrm{mM}$.

After about 5 days of hospitalisation, patients were released from the hospital, and on the same day a third session of blood flow measurement took place. The strict diabetic control regimen was maintained for 6 months. During that time, patients were seen on a monthly basis by their treating physician and glycosylated haemoglobin levels were obtained. Additional sessions of blood flow measurements were held 2 and 6 months after the institution of strict diabetic control.

Standard seven field stereo colour fundus photographs and fluorescein angiograms were obtained according to the Early Treatment of Diabetic Retinopathy Study (ETDRS) ${ }^{14}$ protocol at baseline and at the end of the 6 months of the study. Assessment of retinopathy was performed in a masked fashion at the Fundus Photographic Reading Center of the University of Wisconsin. An overall retinopathy level according to the ETDRS grading protocol was assigned to the study eye of each patient at baseline and at the end of the study.

Mean brachial artery blood pressure $\left(\mathrm{BP}_{\mathrm{m}}\right)$ was calculated as $\mathrm{BP}_{\mathrm{d}}+1 / 3\left(\mathrm{BP}_{\mathrm{s}}-\mathrm{BP}_{\mathrm{d}}\right)$, where $B P_{s}$ and $B P_{d}$ are the brachial artery systolic and diastolic pressures. Perfusion pressure (PP) was calculated as $2 / 3 \mathrm{BP}_{\mathrm{m}}-\mathrm{IOP}$.

One way analysis of variance and covariance for repeated measures were used in the evaluation of the results. Subsequent analysis of contrast was performed to identify the time at which significant changes occurred. Paired and unpaired Student's $t$ test, correlation and rank correlation analyses were also used in the evaluation of the data. The Wilk-Shapiro test was used to assess the normal distribution of the results. Values of $p<0.05$ were considered statistically significant.

\section{Results}

Institution of strict diabetic control resulted in a significant improvement in average glycosylated haemoglobin (baseline: 11.8\% (2.4\%); month 1 : $10 \cdot 4 \%(1 \cdot 3 \%)$; month $2: 9 \cdot 6 \%(1 \%)$; month 3: $9 \cdot 1 \%(1 \cdot 4 \%)$; month $4: 9 \cdot 6 \%$ $(1.5 \%)$; month 5 : $9.3 \%(1.2 \%)$; and month 6 : $8.9 \%(1 \cdot 6 \%)$. Analysis of variance for repeated measures, $p=0.0001)$. A comparison of fundus photographs taken at the beginning and at the end of the study showed progression of retinopathy by one or more retinopathy levels in eight eyes, and no progression of retinopathy in 20 eyes.

\section{BFS RESULTS}

Average baseline Vleuc, Dens, and Vleuc $\times$ Dens in diabetic subjects $(0.91(0.27)$ $\mathrm{mm} / \mathrm{s}, 198$ (146), and 173 (132) AU, respectively) were not significantly different from those of normal subjects $(0.97(0.17) \mathrm{mm} / \mathrm{s}$, 135 (63), and 131 (68) AU, respectively, nonpaired Student's $t$ test).

A one way analysis of variance for repeated measures showed a significant increase in Vleuc in time $(p=0.013$, Fig 1). A subsequent analysis of contrast performed in order to determine the time at which this significant change occurred showed an average $16 \%$ increase from baseline at 5 days $(p=0.03), 12 \%$ increase from baseline at 2 months $(p=0.08)$, and $15 \%$ increase from baseline at 6 months $(p=0.03)$. No significant differences in the changes in Vleuc over time were observed between eyes that had progression or no progression of retinopathy $(p=0 \cdot 27)$. Although during the study, eyes with progression of retinopathy had a tendency towards higher Vleuc values than eyes without progression, the difference was not statistically significant $(p=0.09)$.

During the whole of the study, no significant changes in time nor differences between eyes with and eyes without retinopathy progression were detected in Dens, the number of entoptically observed leucocytes per field (one way analysis of variance). With the variability present in our Dens measurements, we would have been able to detect an average change in Dens of $30 \%$ or more with a $66 \%$ power. 

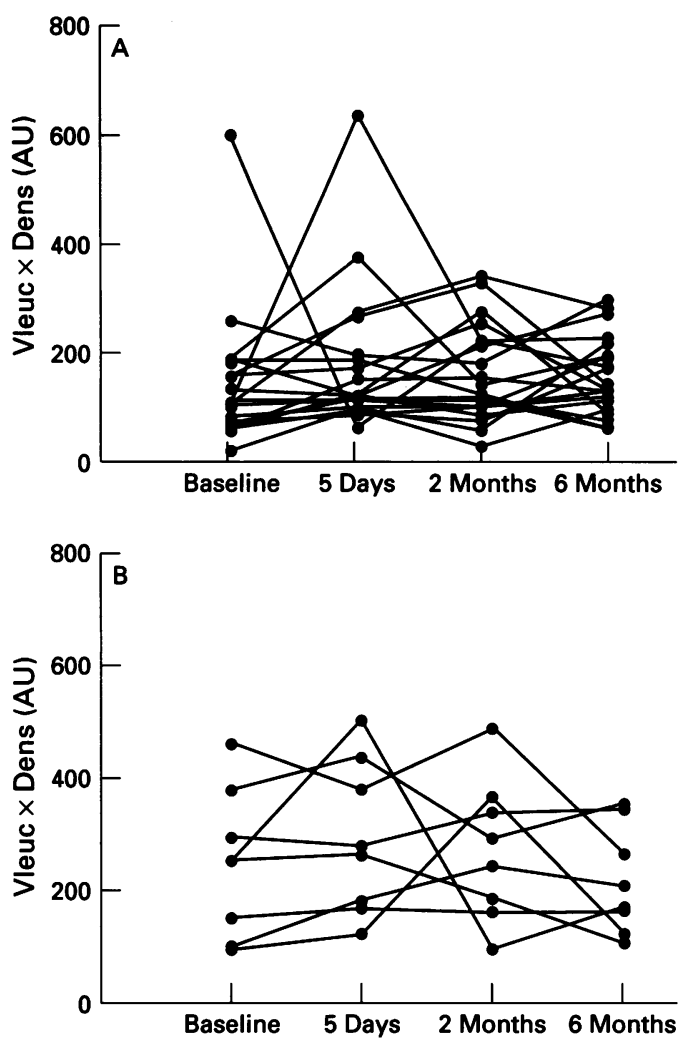

Figure 2 Product of leucocyte velocity and density (Veuc $\times$ Dens) in the retinal macular circulation at baseline and then 5 days, 2 months, and 6 months after the institution of strict glycaemic control. (A) Eyes that showed no progression of retinopathy at the end of the 6 months study. (B) Eyes that showed progression of retinopathy at the end of the study.

The product Vleuc $\times$ Dens, however, was significantly higher in eyes with progression of retinopathy than in eyes without progression of retinopathy during the whole of the study (one way analysis of variance, $p=0.005$, Fig 2 ). No significant changes over time, however, were observed in Vleuc $\times$ Dens $(p=0.47)$ in eyes with and without progression of retinopathy. Furthermore, although the average product of Vleuc $\times$ Dens at baseline for the whole group of diabetic patients was not significantly higher than normal, the value for the eight eyes that showed progression of retinopathy (251 (130) $\mathrm{AU}$ ) was about $92 \%$ higher than normal (131 (68) AU, non-paired Student's $t$ test, $\mathrm{p}<0.05$ ).

The regulatory response to hyperoxia, $R_{\text {Vleuc }}$, was also not affected by the institution of strict diabetic control and no significant differences were observed between eyes with or without progression of retinopathy.

No significant associations were detected by one way analysis of covariance between Vleuc, Dens, or Vleuc $\times$ Dens and: (1) glycosylated haemoglobin, (2) blood glucose measured at the time of flow determination, and (3) the average of blood glucose measurements obtained during the 4 days preceding flow determinations.

Correlation analysis of these variables for individual measurement sessions showed a statistically significant association between glycosylated haemoglobin and Vleuc $\times$ Dens only at baseline flow measurements $(r=-0.4$, $\mathrm{p}=0.034$, Fig 3). No significant correlations were observed between Vleuc or Vleuc $\times$ Dens and retinopathy level or length of diabetes mellitus.

\section{COMPARISON OF BFS WITH BLDV AND MFP RESULTS}

No significant correlation was found between measurements of Vleuc in the macular circulation and measurements of $Q$ in major retinal vessels. Also, no significant correlations between relative changes from baseline in Vleuc and $Q$ were observed on the fifth day of strict diabetic control $(r=0 \cdot 13, \mathrm{p}=0.49)$. Statistically significant correlations between these variables, however, were present at 2 months $(r=0.49, \mathrm{p}=0.008)$ and at 6 months $(r=0.58, \mathrm{p}=0.001)$. Dens or Vleuc $\times$ Dens did not correlate with any measurements obtained by BLDV and MFP.

The average regulatory response to hyperoxia $R_{Q}(-46 \cdot 6 \%(7 \cdot 9 \%))$ was significantly larger than the average responses $\mathbf{R}_{\text {Vleuc }}$ $\left(-12 \cdot 2 \%(13 \cdot 3 \%)\right.$ and $R_{\text {Vleuc } \times \text { Dens }}(-25 \cdot 5 \%$ $(17 \cdot 8 \%)$ ) (non-paired Student's $t$ test, $\mathrm{p}<0.001$ and $\mathrm{p}<0.001$, respectively). No significant associations were detected during the study between $R_{Q}$ and $R_{\text {Vleuc }}$ and between $R_{Q}$ and $R_{\text {Vleuc } \times \text { Dens (one way analysis of }}$ covariance).

\section{Discussion}

During the length of our study, subjects showed a significant improvement in glycaemic control, which was similar to that obtained in the strict diabetic control group of the DCCT study. ${ }^{1}$ Improved glycaemic control was associated with a significant increase in Vleuc over time in the macular capillaries, starting from an average of $0.91(0.3) \mathrm{mm} / \mathrm{s}$ at baseline to $1.06(0.3) \mathrm{mm} / \mathrm{s}$ at the end of the study. Although there was an increase in Vleuc during the study, at no time were the average Vleuc values measured significantly different from those present in normal subjects at baseline.

This increase in Vleuc during the 6 months of the study is somewhat surprising because previous studies of the acute effects of a sudden glucose normalisation had shown decreases in flow in large retinal vessels ${ }^{15}$ or no significant

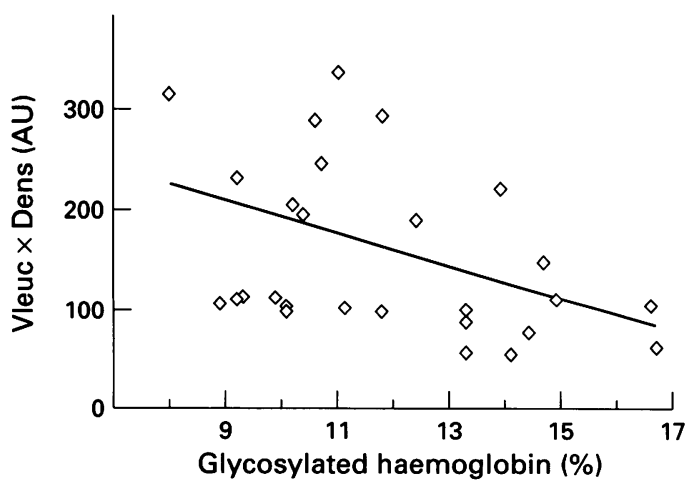

Figure 3 Correlation between glycosylated haemoglobin $(\mathrm{GHb})$ and the product of leucocyte velocity and density (Veuc $\times$ Dens) at baseline. (Correlation coefficient $=-0.4$, $p=0.034$. Regression line equation,

Vleuc $\times$ Dens $=354-15 \cdot 8 \mathrm{GHb}$ ). 
change in the macular microcirculation. ${ }^{16}$ It is possible, however, that the long term effect of more normal glucose levels on retinal capillary flow is different from the acute effect of glucose normalisation. Long term improvement of glucose levels produced by the institution of strict diabetic control could produce metabolic, rheological, or other changes leading to an increase in Vleuc. For example, changes in leucocyte rigidity and adhesiveness, which can occur with improved diabetic glycaemic control could also affect BFS measurements and lead to differences between flow changes determined in large retinal vessels and those observed in the macular capillaries.

Although our results showed a mild increase in time in Vleuc of about $15 \%$ in the diabetic patients under strict glycaemic control, Vleuc in these patients was not significantly different from normal, a finding that is somewhat different from the reports of Sinclair ${ }^{17}$ and Fallon et al $^{18}$ who found an increase in Vleuc in diabetic patients. Differences in patient selection could account, in part, for this discrepancy. Sinclair et $a l,{ }^{17}$ for example, included diabetic patients under 'stable metabolic control' whereas our study was performed in poorly controlled subjects. Fallon et al ${ }^{18}$ included in their study older and probably non-insulin dependent diabetic patients with higher systemic blood pressures and these factors could perhaps account for some of the differences seen between studies. ${ }^{819}$

Our Vleuc results are also different from those reported by Arend et $a l,{ }^{20}$ who studied diabetic patients with average glycaemic control and compared them with normals using the scanning laser technology with fluorescein angiography. They showed, in diabetic patients, decreased capillary blood flow velocity in perifoveal capillaries. Differences in patient selection could again account, in part, for the differences observed since our current study shows, for example, that long term improvement of glycaemia results in an increase in Vleuc in the macular circulation.

Differences in the techniques used could also account for some of the discrepancy. Arend et $a l^{20}$ determined the velocities of segments of low and high fluorescence observed in retinal perifoveal capillaries close to the foveal avascular zone during fluorescein angiography. These segments may not be the same as the bright particles observed on the blue field simulation technique entoptic phenomenon which are thought to correspond to leucocytes. ${ }^{21}$ The retinal capillaries in the macula are arranged in a multilayered fashion and it is also possible that the entoptically observed particles may be produced by leucocytes moving at a certain layer whereas the segments seen in fluorescein angiography may correspond to flow in different layers. These arguments are supported by the fact that absolute capillary velocities reported by Arend et $a l^{20}$ in healthy volunteers $(3.28(0.45)$ $\mathrm{mm} / \mathrm{s}$ ) are more than three times higher than those seen in our study.

Vleuc represents leucocyte velocity in the retinal macular capillaries. In these capillaries of a diameter of 7 to $10 \mu \mathrm{m}$ the speed of leucocytes is very close to the mean speed of bulk flow. ${ }^{22}$ Changes in Vleuc would represent changes in blood flow if we can assume that capillary diameter remains unchanged throughout the study. As discussed by Riva et $a l^{23}$ the constancy of capillary diameter is supported by work of Friedman et $a l^{24}$ who found no evidence of retinal capillary vasomotion, by work of Baez et al ${ }^{25}$ who showed that capillary diameter remains constant within a wide range of perfusion pressures and by theoretical considerations on the structural rigidity of capillaries. ${ }^{26}$

Closure of retinal capillaries, however, has been described in diabetic retinopathy. ${ }^{27}$ In addition, Ben-Nun et $a^{28}$ have shown that in diabetic rats, retinal capillaries can be open to plasma flow but closed to the passage of erythrocytes. Because of these pathological mechanisms it is possible that in diabetic retinopathy changes in Vleuc may not accurately represent changes in blood flow in the macular area. In an attempt to account for the possibility that capillaries may become occluded in diabetic retinopathy, we also measured Dens, the number of entoptically observed leucocytes per field and we calculated the product Vleuc $\times$ Dens.

Average Vleuc $\times$ Dens was significantly higher than normal in eyes with retinopathy progression. This variable was also significantly higher in eyes that showed progression of retinopathy than in eyes with no progression, suggesting that macular blood flow is higher in eyes that show progression of retinopathy. These results fit well with those of Brinchmann-Hansen et $a^{29}$ who reported that eyes that show progression of retinopathy after institution of strict diabetic control have larger retinal vessel diameters than those of eyes that don't progress, thus suggesting that blood flow may also be larger in these eyes. They also support the haemodynamic hypothesis ${ }^{30} 31$ which postulates that increased blood flow through a vascular bed may play a role in the development of important diabetic microangiopathic features such as increased vascular permeability and capillary closure.

Significant correlations between changes in Vleuc measured in the macula and changes in $Q$ obtained in the large vessels were present at 2 and 6 months and not at 5 days of strict diabetic control. These results suggest that improvement of glycaemic levels may result in better correlation between blood flows in these two sites. This effect could be due in part to the improvement in retinal vascular regulatory responses which has been reported in humans ${ }^{15}$ and dogs ${ }^{32}$ when high glucose levels are lowered towards normal. This increased correlation was observed only after 2 months and 6 months of improved glycaemic control, suggesting an effect that becomes evident after a prolonged improvement of glycaemia.

Although we found some associations between relative changes in $Q$ and Vleuc, BFS results were somewhat different from those observed by BLDV veins. For example, Vleuc $\times$ Dens was significantly larger than 
normal in eyes with progression of retinopathy. In these eyes, this variable was also larger than that of eyes without progression throughout the length of the study $(p=0 \cdot 0055)$. As we have reported previously, $Q$ measurements obtained in the large retinal veins, on the other hand, showed no significant differences between eyes with and without progression of retinopathy. 89

In addition, we have also reported that in the main retinal veins there were changes in $Q$ from baseline on the fifth day of strict glycaemic control that were different in eyes without and with progression of retinopathy. ${ }^{89}$ Eyes without progression showed a significant average decrease in $Q$ on the fifth day, whereas most eyes with progression showed increases in Q. Furthermore, the magnitude of the individual $\mathrm{Q}$ changes observed on the fifth day correlated significantly with the amount of change in retinopathy level. No similar changes were observed in the macular microcirculation.

The macula is a specialised part of the retina and it is possible that the changes occurring in its circulation may differ from those occurring in the more peripheral retina. One example of such discrepancy is suggested by the different responses of the peripheral and macular retinal circulations to hyperglycaemia and a sudden reduction in blood glucose. Grunwald et al ${ }^{15}$ using BLDV, reported that $Q$ in the main retinal veins was higher than normal during hyperglycaemia in poorly controlled diabetics. A sudden normalisation of blood glucose by insulin administration resulted in a significant decrease in Q. Fallon et $a^{33}$ and Davies et al, ${ }^{16}$ using BFS, on the other hand, did not detect any significant effects of acute hyperglycaemia and reduction in plasma glucose on leucocyte velocity in the macular capillaries.

The regulatory changes in Vleuc and Vleuc $\times$ Dens in response to hyperoxia $\left(R_{\text {Vleuc }}\right.$ and $\left.R_{\text {Vleuc } \times \text { Dens }}\right)$ were significantly smaller than the regulatory changes in $Q\left(R_{Q}\right)$, a result that is in accordance with those reported by Petrig et $a l^{34} 35$ and Sponsel et al. ${ }^{36}$ In addition, no correlation was found between the vascular regulatory responses measured in large retinal veins and those determined in the macular microcirculation, a result that further supports the notion that the macular retina may have different metabolic requirements resulting in different blood flow regulatory characteristics.

This lack of significant correlation between the macula and more peripheral retinal circulations could also be due to the fact that measurements of the regulatory responses to hyperoxia using BFS were done in a separate experiment more than 1 hour apart from the experiment in which BLDV and MFP determinations were obtained. Differences in the experimental conditions such as, for example, blood glucose, respiratory volume, and rate and $\mathrm{PCO}_{2}$ between both experiments could perhaps account in part for the lack of correlation between the responses measured by these methods.

Our results also show a weak correlation between glycosylated haemoglobin and
Vleuc $\times$ Dens at baseline before the institution of strict diabetic control. Patients with lower glycosylated haemoglobin tended to have higher Vleuc $\times$ Dens (Fig 3) thus supporting our other results showing that improved glycaemic control produces an increase in Vleuc. These results also suggest that blood glycaemia probably influences both the circulation in the microvasculature of the macula and the large retinal vessels, ${ }^{15}$ a factor that needs to be considered when comparing results of studies performed on patients with different degrees of diabetic glycaemic control.

In summary, institution of strict glycaemic control produces changes in both the circulations of the major retinal veins and the macular capillaries. Although there is an overall association between these beds, there are haemodynamic differences that need to be further investigated in order to shed some light on pathogenetic mechanisms. This investigation was supported by National Institutes of Health (NIH) Grant EY05775, the Vivian Simkins Lasko
Research Fund, and assisted by Clino Data Management and Research Fund, and assisted by Clino Data Management and
analysis provided by Grant RR-00040 from NIH. Proprietary analysis provided by Grant RR-00040 from NIH. Proprietary equipment used in this study. Other authors have no proprietary interest in the development or marketing of this or a competing instrument.

The authors thank Louise Epperson for her significant help in the performance of this study and Dolly Scott for the preparation of the manuscript.

1 The Diabetes Control and Complication Trial Research Group. The effect of intensive treatment of diabetes on the development and progression of long term complications in insulin dependent diabetes mellitus. $N$ Engl $\mathfrak{f} M e d$ 1993; 329: 977-86.

2 Lauritzen T, Frost-Larsen $\mathrm{K}$, Larsen HW, Deckert $T$. Effect of 1 year of near-normal blood glucose levels on retinopathy in insulin-dependent diabetics. Lancet 1983; retinopathy $200-4$.

3 Dahl-Jorgensen $\mathrm{K}$, Brinchmann-Hansen $\mathrm{O}$, Hanssen $\mathrm{KF}$, Sandvik L, Aagenaes $O$. Rapid tightening of blood glucose control leads to transient deterioration of retinopathy in insulin dependent diabetes mellitus: the Oslo study. $B M \mathcal{F}$ 1985; 290: 811-5.

4 Brinchmann-Hansen O, Dahl-Jorgensen KD, Hanssen KF, Sandvik L, the Oslo Study Group. Effects of intensified insulin treatment on various lesions of diabetic retinopathy. Am $\mathcal{f}$ Ophthalmol 1985; 100: 644-53.

5 Dahl-Jorgensen $\mathrm{K}$, Brinchmann-Hansen $\mathrm{O}$, Hanssen $\mathrm{KF}$. Effect of near normoglycemia for two years on progression of early diabetic retinopathy, nephropathy and neuroof early diabetic retinopathy, nephropathy and
pathy: the Oslo Study. $B M \mathcal{F}$ 1986; 293: 1195-9.

6 The DCCT Research Group. Are continuing studies of metabolic control and microvascular complication in insulin-dependent diabetes mellitus justified? $N$ Engl $\mathcal{f}$ Med 1988; 318: 246-50.

7 Brinchmnn-Hansen O, Dahl-Jorgensen K, Hanssen KF, Sanduik $\mathrm{L}$. The response of diabetic retinopathy to 41 months of multiple insulin injection, insulin pumps and conventional insulin injections. Arch Ophthalmol 1988; 106: $1242-6$.

8 Grunwald JE, Brucker AJ, Schwartz SS, Brunstein SN, Baker L, Petrig BL, et al. Diabetic glycemic control and Baker L, Petrig BL, et al. Diabetic glycemic
retinal blood flow. Diabetes 1990; 39: 602-7.

9 Grunwald JE, Brucker AJ, Braunstein SN, Schwartz SS, Baker L, Petrig BL, et al. Strict metabolic control and retinal blood flow in diabetes mellitus. $\mathrm{Br} \mathcal{F}$ Ophthalmol 1994; 78: 598-604.

10 Riva CE, Petrig BL. Blue field entoptic phenomenon and blud velocity in the retinal capillaries. F Optom Soc Am 1980; 70: 1234-8.

11 Riva CE, Petrig BL. Retinal blood flow. Laser doppler velocimetry and blue field simulation technique. In: Masters BR, ed. Noninvasive diagnostic techniques in

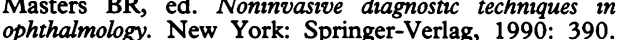
12 Grunwald JE, Riva CE, Sinclair SH, Brucker AJ, Petrig BL. Grunwald JE, Riva CE, Sinclair SH, Brucker AJ, Petrig BL.
Laser Doppler velocimetry study of retinal circulation in Laser Doppler velocimetry study of retinal circulation

13 The DCCT Research Group. Diabetes Control and Complications Trial (DCCT). Results of feasibility study. Diabetes Care 1987; 10: 1-9.

14 Early Treatment Diabetic Retinopathy Study Research Group. Grading diabetic retinopathy from stereoscopic color fundus photographs - an extension of the modified Airlie House Classification. EDTRS Report Number 10. Ophthalmology 1991; 98: 786-806.

15 Grunwald JE, Riva CE, Martin DB, Quint AR, Epsetin PA. Effect of an insulin-induced decrease in blood glucose on the human diabetic retinal circulation. Ophthalmology 1987; 94: 1614-20. 
16 Davies EG, Hyer SL, Kohner EM. Macular blood flow response to acute reduction of plasma glucose in diabetic patients measured by the blue light entoptic technique. Ophthalmology 1990; 97: 160-4.

17 Sinclair SH. Macular retinal capillary hemodynamics in diabetic patients. Ophthalmology 1991; 98: 1580-6.

18 Fallon TJ, Chowiencyzk P, Kohner EM. Measurement of retinal blood flow in diabetes by the blue light entoptic phenomenon. Br f Ophthalmol 1986; 70: 43-6.

19 Grunwald JE, Piltz J, Patel N, Bose S, Riva CE. Effect of aging on retinal macular microcirculation: $A$ blue field aging on retinal macular microcirculation: A blue field
simulation study. Invest Ophthalmol Vis Sci 1993; 34: 3609-13.

20 Arend O, Wolf S, Jung F, Bertram B, Postgens H, Toonen $\mathrm{H}$, et al. Retinal microcirculation in patients with diabetes mellitus: Dynamic and morphological analysis of perifoveal capillary network. Br f Ophthalmol 1991; 75: 514-8.

21 Sinclair SH, Azar-Cavanagh MA, Soper KA, Tuma RF, Mayrovitz HN. Investigation of the source of the blue field entoptic phenomenon. Invest Ophthalmol Vis $\mathrm{Sci}_{\mathrm{C}}$ 1989; 30: 668-73.

22 Schmid-Schonbein GW, Skalak R, Usami S, Chein S. Cell distribution in capillary networks. Microvasc Res 1980; 19: $18-44$

23 Riva CE, Sinclair SH, Grunwald JE. Autoregulation of retinal circulation in response to decrease of perfusion pressure. Invest Ophthalmol Vis Sci 1981; 21: 34-8.

24 Friedman E, Smith TR, Kuwabara T. Retinal microcirculation in vivo. Invest Ophthalmol Vis Sci 1964; 3: 217-26.

25 Baez S, Lamport H, Baez A. Pressure effects in living microscopic vessels. In: Copley AL, Staisby G, eds. Flow properties of blood. London: Pergamon Press, 1960: 122-36.

26 Murphy ME, Johnson PC. Possible contribution of basement membrane to the structural rigidity of blood capillaries. Microvasc Res 1975; 9: 242-5.
27 Kohner EM, Dollery CT, Paterson JW, Oakley MB. Arterial fluorescein studies in diabetic retinopathy. Diabetes 1967; 16: 1-10.

28 Ben-Nun J, Alter VA, Constable IJ, Roberts CE. The patency of the retinal vasculature to erythrocytes in retinal vascular disease. Invest Ophthalmol Vis Sci 1990; 31: 464-70.

29 Brinchman-Hansen O, Dahl-Jorgensen K, Hanssen KF, Sandvik L. Effects of intensified insulin treatment on retinal vessels in diabetic patients. Br $\mathcal{f}$ Ophthalmol 1988; 72: $666-73$.

30 Parving $\mathrm{HH}$, Viberti GC, Keen $\mathrm{H}$, Christiansen JS, Lassen NA. Hemodynamic factors in the genesis of diabetic microangiopathy. Metabolism 1983; 32: 943-9.

31 Zata R, Brenner BM. Pathogenesis of diabetic microangiopathy. The hemodynamic view. Am $\mathcal{F}$ Med 1986; 80 443-53.

32 Ernest JT, Goldstick TK, Engerman RL. Hyperglycemia impairs retinal oxygen autoregulation in normal and diabetic dogs. Invest Ophthalmol Vis Sci 1983; 24: 985-9.

33 Fallon TJ, Sleightholm MA, Merrick C, Chahal MP, Kohner EM. The effect of acute hyperglycemia on flow velocity in the macular capillaries. Invest Ophthalmol Vis Sci 1987; 28: 1027-30.

34 Petrig BL, Riva CE, Sinclair SH, Grunwald JE Quantification of changes in leukocyte velocity in retina macular capillaries during oxygen breathing. Inves Ophthalmol Vis Sci 1982; 22 (suppl): 194.

35 Petrig BL, Riva CE, Grunwald JE, Baine JC. Effect of graded oxygen breathing on macular capillary leukocyte velocity. Invest Ophthalmol Vis Sci 1986; 27 (suppl): 221.

36 Sponsel WE, DePaul KL, Zetlan SR. Retinal hemodynamic effects of carbon dioxide, hyperoxia, and mild hypoxia. Invest Ophthalmol Vis Sci 1992; 33: 1864-9. 